\title{
Nonlinear Optical Study of 1,1'-Methylenedipyridinium bis[tetrachloride gold(III)] Hybrid Salt
}

\author{
M.D. Zidan ${ }^{a, *}$, M.M. Al-Ktaifani ${ }^{b}$ And A. AllahHam ${ }^{a}$ \\ ${ }^{a}$ Department of Physics, Atomic Energy Commission, P.O. Box 6091, Damascus, Syria \\ ${ }^{b}$ Department of Radioisotopes, Atomic Energy Commission, P.O. Box 6091, Damascus, Syria \\ (Received March 1, 2017; revised version May 25, 2017; in final form July 3, 2017)
}

\begin{abstract}
The third-order nonlinear optical properties of 1,1'-methylenedipyridinium bis[tetrachloride gold(III)] were investigated by the $z$-scan method using CW diode laser. The experimental results led to estimation of the nonlinear optical absorption coefficient $(\beta)$, the nonlinear optical refraction $\left(n_{2}\right)$, and third-order nonlinear optical susceptibility $\left(\chi^{3}\right)$. Our results show that the excited-state absorption cross-sections $\left(\sigma_{e x}\right)$ is larger than the groundstate absorption cross-sections $\left(\sigma_{g}\right)$, this suggests that the nonlinear optical absorption in the MPTC gold(III) is due to the reverse saturable absorption mechanism. Furthermore, the obtained results suggest that our material is considered as a promising candidate for future optical devices applications.
\end{abstract}

DOI: 10.12693/APhysPolA.132.1379

PACS/topics: 1,1'-methylenedipyridinium bis[tetrachloride gold (III)], organic-metallic complex $z$-scan technique

\section{Introduction}

A strong motive in searching for new materials with a large third-order nonlinear optical (NLO) coefficient at room temperature comes from the growing need of passive optical limiters, for human sight protection, and optical sensors from hazardous laser beams [1-3]. Among the reported materials are including: organic compounds [46], different organic dyes [7] and $\mathrm{C}_{60}$ derivatives $[8,9]$. But, the organometallic complexes have attracted the researchers for their unique NLO properties [10-13]. These types of substances, which consist of transition metal centers and unsaturated organic ligands have reasonable third-order nonlinear optical properties. These unique properties are due to the richness of the various excited states presented in the system as well as the delocalization of $\pi$-electrons and the transfer of electrons between the metal center and the ligands.

Third-order nonlinear optical coefficient $\left(\chi^{3}\right)$ has been characterized by different techniques [14]. These techniques have their own advantages and disadvantages. Among them, the $z$-scan technique $[15,16]$ has a great advantage, because of its simplicity and high sensitivity by which one can simultaneously characterize the nonlinear optical absorption and the refraction of the nonlinear optical materials. Despite, a number of organometallic complexes have been studied [17-22], using $z$-scan technique, but the investigations of such materials are still unexploited. Very recently, nonlinear optical investigation of 1,1'-methylenedipyridinium tetrachloridecuprate(II)] was reported by us [23]. In this article, we will introduce third-order nonlinear optical properties of the analogous Au complex 1,1'-methylenedipyridinium bis[tetrachloride $\operatorname{gold}(\mathrm{III})]$.

*corresponding author; e-mail: PScientific@aec.org.sy

\section{Experimental techniques}

The studied complex of 1,1'-methylenedipyridinium bis[tetrachloride gold(III)], $\left[\left(\mathrm{C}_{5} \mathrm{H}_{5} \mathrm{~N}\right)_{2} \mathrm{CH}_{2}\right]\left[\mathrm{AuCl}_{4}\right]_{2}$ was prepared and characterized by the procedures described in Ref. [24]. The name of the complex "1,1'methylenedipyridinium bis[tetrachloride gold(III)]" is abbreviated to "MPTC gold(III)".

The $z$-scan experimental setup was analogous to that described in Ref. [6]. The experimental parameters were used as follows: the laser beam was focused by a $10 \mathrm{~cm}$ focal length lens to the radius of $\left(\omega_{0}\right)$ of $34 \mu \mathrm{m}$ at the focal point. The diffraction length (the Rayleigh length) $z_{0}$ was $5.5 \mathrm{~mm}$, the radius of the aperture $\left(r_{a}\right)$ was $0.5 \mathrm{~mm}$ and the radius of the laser beam waist was $\omega_{a}=1 \mathrm{~mm}$ on the aperture. The measurements were carried out with linearly polarized $\mathrm{TEM}_{00}$ Gaussian beam of a CW diode laser at $\lambda=635 \mathrm{~nm}\left(\mathrm{CUBE}^{\mathrm{TM}}\right.$ Diode Laser System, Coherent, 635-3QE). The studied sample was dissolved in dimethyl sulfoxide (DMSO) with a concentration of $10^{3} \mathrm{M}$. The $2 \mathrm{~mm}$ thick quartz cell which was held on translation stage was precisely moved through the focal area of the beam over a length of $4 \mathrm{~cm}$. A power meter (Thorlabs PM300E) was used to measure the reference beam and transmitted beam powers. All the equipment parts were controlled by a computer for further data processing.

\section{Results and discussion}

The UV-vis spectrum of the MPTC gold(III) solution was recorded in the wavelength range $250-800 \mathrm{~nm}$ using UV-3101 PC Shimadzu Spectrophotometer and is depicted with the chemical structure in Fig. 1. The spectrum exhibits two maximum absorption peaks at $265 \mathrm{~nm}$ and $330 \mathrm{~nm}$. They are readily assigned to $\pi \rightarrow \pi^{*}$ transition within the organic dication $\left[\left(\mathrm{C}_{5} \mathrm{H}_{5} \mathrm{~N}\right)_{2} \mathrm{CH}_{2}\right]^{2+}$ and $d \rightarrow d^{*}$ transition of the inorganic ionic fragment $\left[\mathrm{AuCl}_{4}\right]^{-}$, respectively. 


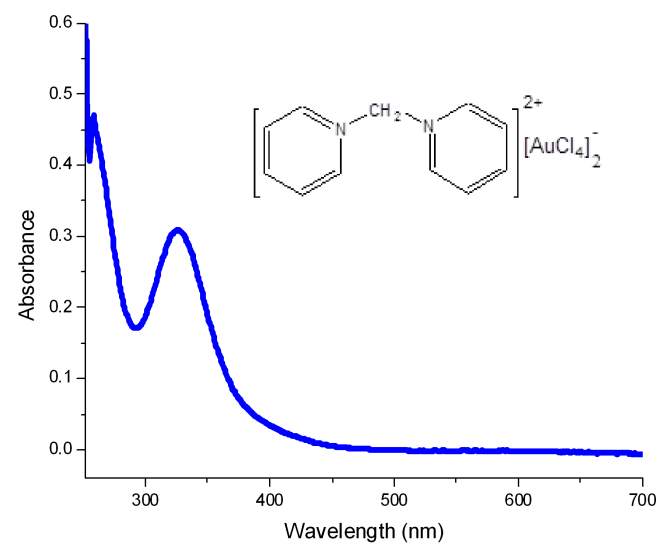

Fig. 1. The chemical structure and the UV-vis spectrum of the MPTC gold(III) in DMSO with concentration of $10^{-3} \mathrm{M}$ in DMSO.

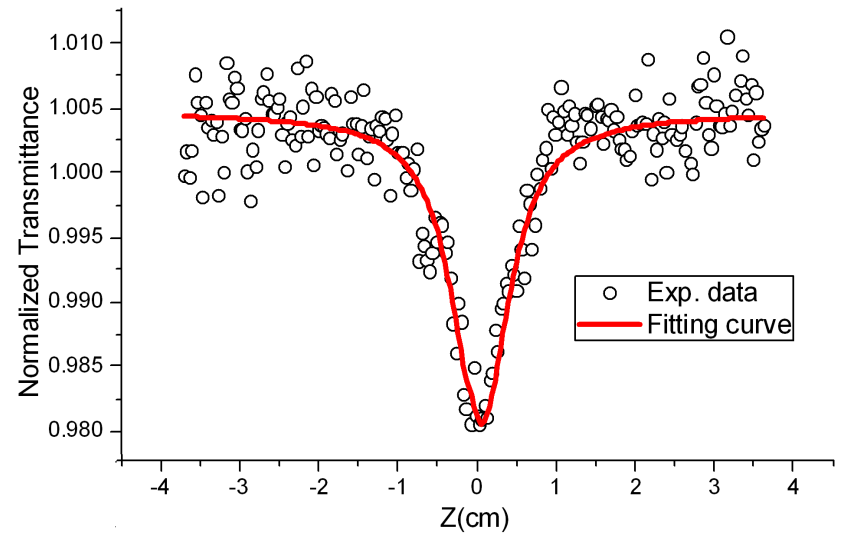

Fig. 2. Open-aperture $z$-scan data of the MPTC gold(III) in DMSO with concentration of $10^{-3} \mathrm{M}$ in DMSO, solid line depict the theoretical fit.

In order to investigate the third order nonlinear optical properties of the MPTC gold(III) in DMSO, the sample was prepared with concentration of $10^{-3} \mathrm{M}$; the laser was set at peak power intensity of $930 \mathrm{~W} / \mathrm{cm}^{2}$.

Figure 2 shows the open-aperture (OA) z-scan measurements of MPTC gold(III) in DMSO. The resulted curve reflects decrease of the transmittance at $z=0$ with increase of the power intensity $I_{0}$. This is the main feature of a reverse saturable absorption (RSA) mechanism according to the literature. A classical five-level model has been proposed to explain the nonlinear optical absorption of organic compounds with the delocalization of $\pi$-electrons system [5, 25-27].

The nonlinear optical absorption coefficient $(\beta)$ is readily obtained from the best fitting performed on the experimental data of the $\mathrm{OA}$ measurements with equation $[15,16]$ :

$$
T(z)=1-\frac{I_{0} L_{e f f} \beta}{2^{\frac{3}{2}}\left(1+\frac{z^{2}}{z_{0}^{2}}\right)} .
$$

In the above Eq. (1), $L_{e f f}=(1-\exp (-\alpha L)) / \alpha$ is the effective thickness of the sample, $L$ is the thickness of the sample, $\alpha$ is the linear optical absorption coefficient, $z_{0}=\pi \omega_{0}^{2} / \lambda$ is diffraction length of the beam, $\lambda$ is the laser wavelength, and $I_{0}=930 \mathrm{~W} / \mathrm{cm}^{2}$ is the laser intensity at $z=0$. Figure 2 shows the fitting curve "solid line", while the "symbols" are the experimental data to the nonlinear optical transmittance given by Eq. (1).

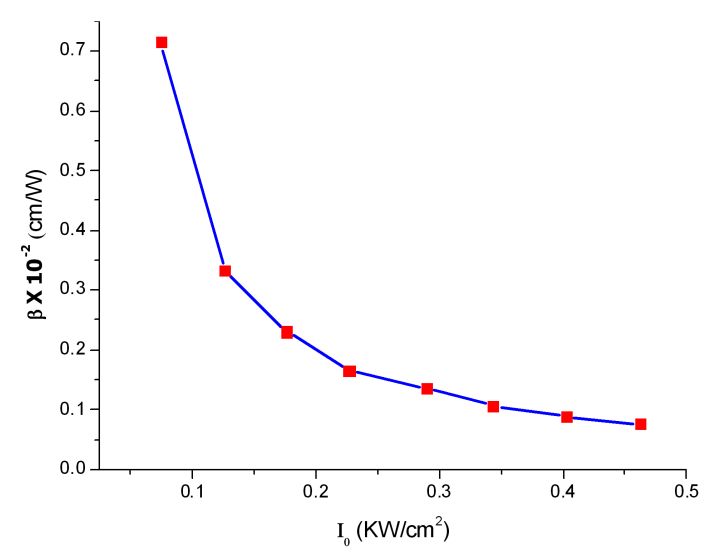

Fig. 3. Nonlinear absorption coefficient $\beta$ versus onaxis input intensity $I_{0}$ of the MPTC gold(III) in DMSO with concentration of $10^{-3} \mathrm{M}$ in DMSO.

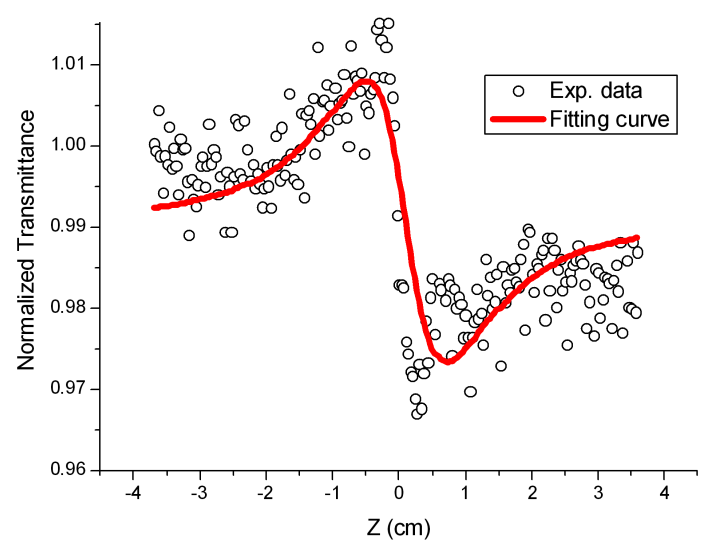

Fig. 4. Pure nonlinear refraction curve of the MPTC gold(III) in DMSO with concentration of $10^{-3} \mathrm{M}$ in DMSO, solid line depict the fitting curve.

The dependence of excitation intensity $I_{0}$ on nonlinear optical absorption coefficients $(\beta)$ is shown in Fig. 3 . It is noticed that the nonlinear optical absorption coefficient $(\beta)$ decreases gradually with increasing excitation intensity, which is a consequence of sequential two-photon absorption. That is, the observed nonlinearity is the result of two photon absorption (TPA) and excited state absorption (ESA) assisted RSA process [25, 28]. This phenomenon is confirmed by comparing the magnitude of the cross-section of the ground state $\left(\sigma_{g}\right)$ and the crosssection of the excited state $\left(\sigma_{e x}\right)$. Equation $(2)$ shows the relation between the optical absorption coefficient $\beta$ and the term $\Delta \sigma[29]$ :

$$
\beta=\frac{\lambda N_{0} \Delta \sigma}{4 \pi I_{s}},
$$

where $\Delta \sigma=\sigma_{e x}-\sigma_{g}, N_{0}$ is the total concentration in 
cubic unit $\left(\mathrm{cm}^{3}\right)$, and $I_{s}=\frac{h c}{\lambda \sigma_{g} \tau}$, where $\frac{h c}{\lambda}$ is the pumpphoton energy, $\tau$ is the excited lifetime and taken to be $1 \mathrm{~ms}$ (triplet state decay time). Both values of $\sigma_{g}$ and $\sigma_{e x}$ were calculated to be $5.03 \times 10^{-19} \mathrm{~cm}^{2}, 7.62 \times 10^{-14} \mathrm{~cm}^{2}$, respectively. The obtained outcome indicates that the $\sigma_{e x} \gg \sigma_{g}$, which strongly suggests that the nonlinearity in the studied sample was mostly associated with RSA in the MPTC gold(III).

To determine the sign and magnitude of nonlinear optical refractive index $\left(n_{2}\right)$ of the MPTC gold(III), a closed aperture (CA) z-scan measurements were performed by placing a small hole in front of detector. The division method (CA/OA) was applied to separate the nonlinear optical refraction from that of the nonlinear optical absorption components. The obtained data reflect purely the effect of nonlinear optical refraction index $\left(n_{2}\right)$. Figure 4 shows the pure nonlinear optical refraction curves of the MPTC gold(III) in DMSO with a concentration of $10^{-3} \mathrm{M}$.

The nonlinear phase shift at the focus $\Delta \varphi$ was obtained through fitting the CA/OA data (Fig. 4) with the following approximate equation $[15,16]$ :

$$
T\left(z, \Delta \varphi_{0}\right)=-\frac{4 \Delta \varphi_{0} X}{\left(X^{2}+9\right)\left(X^{2}+1\right)},
$$

where $X=\left(Z / Z_{0}\right)$, and $T$ is the normalized transmittance for $\mathrm{CA} / \mathrm{OA}$ curve. Then, inserting the value of $\Delta \varphi$ in Eq. (4), one can obtain the value of $n_{2}$ :

$$
n_{2}=\frac{\lambda \Delta \varphi_{0}}{2 \pi I_{0} L_{e f f}} .
$$

The corresponding real and imaginary parts of the thirdorder nonlinear optical susceptibility $\left(\chi^{3}\right)$, can be determined by inserting the values of $n_{2}$ and $\beta$ in Eqs. (5) and (6).

$$
\begin{aligned}
& \operatorname{Re} \chi^{3}[\mathrm{esu}]=\left(10^{-4} \varepsilon c^{2} n^{2} / \pi\right) n_{2}\left[\mathrm{~cm}^{2} / \mathrm{W}\right], \\
& \operatorname{Im} \chi^{3}[\mathrm{esu}]=\left(10^{-2} \varepsilon c^{2} n^{2} \lambda / 4 \pi^{2}\right) \beta[\mathrm{cm} / \mathrm{W}],
\end{aligned}
$$

where $\varepsilon_{0}$ is the vacuum permittivity and $c$ is the speed of light in vacuum.

The values of $\alpha_{0}$ and $n_{0}$ were obtained using similar method described in our previous work [22]. The obtained values of $\alpha_{0}, n_{0}, n_{2}, \beta,\left|\operatorname{Re} \chi^{3}\right|$ and $\left|\operatorname{Im} \chi^{3}\right|$ are listed in Table I.

Using a CW laser can lead to large nonlinear optical effect as result of thermal processes. The nonlinear thermal effect is raised from the absorption of the incident CW laser beam through an absorbing medium. The temperature of the absorbing material consequently increases, which leads to variation of the refractive index of the absorbing material [30].

The thermal nonlinearity $\left(n_{2}\right)$ is related with the thermo-optic coefficient $\left(\frac{\mathrm{d} n}{\mathrm{~d} T}\right)$ by Eq. (7) [30]:

$$
n_{2}^{(t h)}=\left(\frac{\mathrm{d} n}{\mathrm{~d} T}\right) \frac{\alpha_{0} \omega_{0}^{2}}{\kappa},
$$

where $\kappa=0.1567 \mathrm{~W} /(\mathrm{m} \mathrm{K})$ is the thermal conductivity of the DMSO solvent and $\omega_{0}=34 \mu \mathrm{m}$ is the radius of the laser beam at the focal point. Equation (7) indicates that the $n_{2}^{(t h)}$ quantity is geometry-dependent through the $\omega_{0}$ factor and is not an intrinsic property of an optical material (more discussion on the thermal nonlinearities with CW laser beams can be found in Ref. [30]). Using Eq. (7), the value of the thermo-optic coefficient $\left(\frac{\mathrm{d} n}{\mathrm{~d} T}\right)$ of the MPTC gold(III) in DMSO has been found to be $\left.9.96 \times 10^{-6} \kappa^{-1}\right)$.

The curve in Fig. 4 exhibits pre-focal peak and post-focal valley configuration, which is direct indication of negative $n_{2}$ (negative lens) and our sample MPTC gold(III) is also considered being self-defocusing material around $635 \mathrm{~nm}$. The defocusing effect is due to the thermal effect arising from the process of absorbing laser radiation then the non-radiative relaxation of the excited states makes the local temperature of the studied medium to be high. This suggests that the absorbing medium acts as a thermal lens. In other word, there will be severe phase distortion of the propagating beam [29, 31, 32].

It is obvious that our sample shows reasonable thirdorder nonlinear optical properties due to the delocalized electronic states formed between $\pi \rightarrow \pi^{*}$ and $d \rightarrow d^{*}$ transitions, indicating that this type of molecules should be considered as promising NLO materials for future optical devices applications [19].

Our reported values of $n_{2}$ and $\beta$ of the MPTC gold(III) complex listed in Table I are consistent with recently reported result in the literature using continuous wavelength laser [22, 33-37].

To check out the effect of the pure solvent on the recorded result at similar experimental conditions, there was found no significant contribution on the recorded data.

TABLE I

The linear and nonlinear optical parameters of the MPTC gold (III) in DMSO with concentration of $10^{-3} \mathrm{M}$.

\begin{tabular}{c|c|c|c|c|c}
\hline \hline $\begin{array}{c}\alpha_{0} \\
{\left[\mathrm{~cm}^{-1}\right]}\end{array}$ & $n_{0}$ & $\begin{array}{c}n_{2} \times 10^{-9} \\
{\left[\mathrm{~cm}^{2} / \mathrm{W}\right]}\end{array}$ & $\begin{array}{c}\beta \times 10^{-4} \\
{[\mathrm{~cm} / \mathrm{W}]}\end{array}$ & $\begin{array}{c}\operatorname{Re}\left(\chi^{3}\right) \times 10^{-7} \\
{[\mathrm{esu}]}\end{array}$ & $\begin{array}{c}\operatorname{Im}\left(\chi^{3}\right) \times 10^{-5} \\
{[\mathrm{esu}]}\end{array}$ \\
\hline 0.3028 & 1.478 & 5.4 & 3.73 & 3.03 & 1.06
\end{tabular}

\section{Conclusion}

We have demonstrated the $z$-scan measurements of the organometallic complex of the MPTC gold(III) in DMSO with concentration of $10^{-3} M$ using a CW diode laser at $635 \mathrm{~nm}$ wavelength. According to the experimental results, values of $\alpha_{0}, n_{0}, n_{2}, \beta, \operatorname{Re} \chi^{3}, \operatorname{Im} \chi^{3}$ and the thermo-optic coefficient were estimated, and they are affected by the nature of attached ligand. As expected, in the organometallic complex such as: the MPTC gold(III) in DMSO solution, the combination of the organic fragment 1,1'-methylenedipyridinium (with delocalized $\pi$-electron system) and the inorganic part of $\mathrm{Au}$ (III) (with $d$ orbitals) led to a relatively typical optical nonlinearity property.

\section{Acknowledgments}

Our thanks go to Prof. I. Othman, Director General, Prof. M.K. Sabra, and Prof. A.H. Al-Rayyes for their encouragement and support. 


\section{References}

[1] C.S. Suchand Sandeep, A.K. Samal, T. Pardeep, R. Philip, Chem. Phys. Lett. 485, 326 (2010).

[2] B.Y. Xu, Z. Liu, X. Zhang, Y. Wang, J. Tian, Y. Huang, Y. Ma, X. Zhang, Y. Chen, Adv. Mater. 21, 1275 (2009).

[3] J. Wang, W.J. Blau, J. Opt. A Pure Appl. Opt. 11, 024001 (2009).

[4] B. Chandrakantha, A.M. Isloor, R. Philip, M. Mohesh, P. Shetty, A.M. Vijesh, Bull. Mater. Sci. 34, 887 (2011).

[5] M.D. Zidan, A. Arfan, A. Allahham, Opt. Laser Technol. 86, 79 (2016).

[6] M.D. Zidan, M. Al-Ktaifani, A. Allahham, Opt. Laser Technol. 70, 45 (2015).

[7] U.M. Parvin, M.B. Ahamed, Optik 126, 551 (2015).

[8] H.I. Elim, J. Ouyang, J. He, S.H. Goh, S.H. Tang, W. Ji, Chem. Phys. Lett. 369, 281 (2003).

[9] A.R. Kost, J.E. Jensen, R.O. Loufty, J.C. Wither, Appl. Phys. B 80, 281 (2005).

[10] Yun-Dong Zhang, Zhen-Yu Zhao, Cheng-Bao Yao, Lan Yang, Jin Li, Ping Yuan, Opt. Laser Technol. 58, 207 (2014)

[11] M. Saravanan, T.C. Sabari Girisun, G. Vinitha, J. Mater. Sci. 51, 3289 (2016).

[12] S. Mirershadi, S. Ahmadi-Kandjani, A. Zawadzka, H. Rouhbakhsh, B. Sahraoui, Chem. Phys. Lett. 647, 7 (2016).

[13] Cheng-Bao Yao, Yun-Dong Zhang, Hai-Tao Yin, Qing-Yu Meng, Chang-Qiu Yu, Jin Li, Ping Yuan, Chem. Phys. Lett. 576, 35 (2013).

[14] R.L. Sutherlands, Handbook of Nonlinear Optics, Marcel Dekker, New York 2003.

[15] M. Sheik-Bahae, A.A. Said, E.W. Van Stryland, Opt. Lett. 14, 955 (1989).

[16] M. Sheik-Bahae, A.A. Said, T. Wei, D.J. Hagan, E.W. Van Stryland, IEEE J. Quantum Electron. QE-26, 760 (1990).

[17] K.B. Manjunatha, R. Dileep, G. Umesh, B. Ramachandra, Mater. Lett. 105, 173 (2013).

[18] T.C. Sabari Girisun,S. Dhanuskodi, G. Vinitha, Mater. Chem. Phys. 129, 9 (2011).

[19] H. Fan, Q. Ren, X. Wang, T. Li, J. Sun, G. Zhang, D. Xu, G. Yu, Z. Sun, Nat. Sci. 1, 136 (2009).
[20] Ming Kong, Yanqiu Liu, Hui Wang, Junshan Luo, Dandan Li, Shengyi Zhang, Shengli Li, Jieying Wu, Yupeng Tian, Spectrochim. Acta A Mol. Biomol. Spectrosc. 135, 521 (2015).

[21] S. Zongo, M.S. Dhlamini, P.H. Neethling, A. Yao, M. Maaza, B. Sahraoui, Opt. Mater. 50, 138 (2015).

[22] M.D. Zidan, M. Alktaifani, A. Allahham, Optik 126, 1491 (2015)

[23] M.D. Zidan, M. Alktaifani, A. Allahham, Optik 127, 4443 (2016).

[24] M. Al-Ktaifani, M.K. Rukiah, Acta. Crystallogr. C 68, 246 (2012).

[25] V. Singh, P. Aghamkar, B. Lal, Acta Phys. Pol. A 123, 39 (2013).

[26] P. Lind, M.Sc. Thesis, Sweden, Umeå University, 2007.

[27] N.K. Siji Narendran, Rahul Soman, Chellaiah Arunkumar, K. Chandrasekharan, Spectrochim. Acta A Mol. Biomol. Spectrosc. 136, 838 (2015).

[28] S. Pramodini, P. Poornesh, Opt. Laser Technol. 62 , 12 (2014).

[29] F.Z. Henari, P.S. Patil, Opt. Photo. J. 4, 182 (2014).

[30] R.W. Boyd, Nonlinear Optics, 3rd ed., Academic Press, New York 2008.

[31] S. Zafar, Z.H. Khan, M.S. Khan, Spectrochim. Acta A Mol. Biomol. Spectrosc. 114, 164 (2013).

[32] F.Z. Henari, W.J. Blau, Proc. SPIE 2854, 174 (1996).

[33] S. Pramodini, P. Poornesh, Y.N. Sudhakar, M.S. Kumar, Opt. Commun. 293, 125 (2013).

[34] I. Papagiannouli, K. Iliopoulos, D. Gindre, B. Sahraoui, O. Krupka, V. Smokal, A. Kolendo, S. Couris, Chem. Phys. Lett. 554, 107 , (2012).

[35] K. Iliopoulos, I. Guezguez, A.P. Kerasidou, A. ElGhayoury, D. Branzea, G. Nita, N. Avarvari, H. Belmabrouk, S. Couris, B. Sahraoui, Dyes Pigm. 101, 229 (2014).

[36] M.D. Zidan, A.W. Allaf, A. Allahham, A. Al-Zier, Chin. Phys. B 26, 044205 (2017).

[37] K. Iliopoulos, A. El-Ghayoury, B. Derkowska, A. Ranganathan, P. Batail, D. Gindre, B. Sahraoui, Appl. Phys. Lett. 101, 261105 (2012). 